Pacific

Journal of

Mathematics

THE PERIODICITY OF THE SIGNS OF THE COEFFICIENTS OF CERTAIN INFINITE PRODUCTS

Song Heng Chan and Hamza Yesilyurt 


\title{
THE PERIODICITY OF THE SIGNS OF THE COEFFICIENTS OF CERTAIN INFINITE PRODUCTS
}

\author{
Song Heng Chan AND Hamza YesilyuRT
}

\begin{abstract}
Ramanujan, Richmond and Szekeres, Ramanathan, Andrews, Hirschhorn, Alladi and Berndt, and Bressoud have studied certain infinite products arising from continued fractions from the point of view of the periodicity of the signs of their Taylor series coefficients. In this paper we prove more general theorems of the same sort.
\end{abstract}

\section{Introduction}

Recall the definition of the Rogers-Ramanujan continued fraction and its product representation [Hardy and Wright 1960, p. 294],

$$
R(q):=1+\frac{q}{1}+\frac{q^{2}}{1}+\frac{q^{3}}{1}+\cdots=\frac{\left(q^{2} ; q^{5}\right)_{\infty}\left(q^{3} ; q^{5}\right)_{\infty}}{\left(q ; q^{5}\right)_{\infty}\left(q^{4} ; q^{5}\right)_{\infty}} .
$$

Here, we use the familiar notation

$$
(a ; q)_{\infty}=\prod_{n=0}^{\infty}\left(1-a q^{n}\right), \quad|q|<1 .
$$

B. Richmond and G. Szekeres [1978] examined asymptotically the power series coefficients of a large class of infinite products including (1). In particular, if

$$
R(q):=\sum_{n=0}^{\infty} \alpha_{n} q^{n},
$$

they proved that, for $n$ sufficiently large,

$$
\alpha_{5 n}, \alpha_{5 n+1}>0, \quad \text { and } \quad \alpha_{5 n+2}, \alpha_{5 n+3}, \alpha_{5 n+4}<0 .
$$

A similar result was also shown for the coefficients of $1 / R(q)$.

Ramanujan, in his lost notebook [1988], recorded formulas for $\sum_{n=0}^{\infty} \alpha_{5 n+j} q^{n}$, $0 \leq j \leq 4$, which were first proved by G. E. Andrews [1981]. Andrews then used these representations and a theorem of B. Gordon [1961], giving partition-theoretic interpretations of these coefficients, to prove that (2) holds for all $n$, except that

MSC2000: 30B70.

Keywords: infinite products, periodicity, continued fractions. 
$\alpha_{2}=\alpha_{4}=\alpha_{9}=0$. Hirschhorn [1998] later gave a simpler proof of (2) using only the Quintuple Product Identity.

Using the notation of [Andrews and Bressoud 1979], define for $1 \leq r, s<m$

$$
F_{m, r, s}:=F_{m, r, s}(q)=\prod_{n=0}^{\infty} \frac{\left(1-q^{m n+r}\right)\left(1-q^{m n+m-r}\right)}{\left(1-q^{m n+s}\right)\left(1-q^{m n+m-s}\right)}=\frac{\left(q^{r} ; q^{m}\right)_{\infty}\left(q^{m-r} ; q^{m}\right)_{\infty}}{\left(q^{s} ; q^{m}\right)_{\infty}\left(q^{m-s} ; q^{m}\right)_{\infty}}
$$

Throughout the paper we will assume that $\operatorname{gcd}(s, m)=1$ and $s<m / 2$. For $m=5$, $r=2$ and $s=1$, we obtain the product representation of (1). A general result in this direction was established by K. G. Ramanathan [1988], who proved:

Theorem 1.1. Suppose $\operatorname{gcd}(m, r)=1$. Let

$$
F_{m, 2 r, r}:=\sum_{n=0}^{\infty} c_{n} q^{n}
$$

If $\operatorname{gcd}(m, 6)=1$, the signs of the $c_{n}$ 's are periodic with period $m$.

In Section 5, we use Hirschhorn's ideas [1998] in a more general setting to provide a new proof of Theorem 1.1 without the restriction $\operatorname{gcd}(m, 6)=1$.

Define the coefficients $c_{n}$ and $d_{n}$ by

$$
F_{8,3,1}=\sum_{n=0}^{\infty} c_{n} q^{n} \quad \text { and } \quad F_{8,1,3}=\sum_{n=0}^{\infty} d_{n} q^{n}
$$

Gordon's continued fraction has the representation $F_{8,3,1}$. Richmond and Szekeres [1978] proved that $c_{4 n+3}=0$ and $d_{4 n+2}=0$ for all $n \geq 0$. M. D. Hirschhorn [2001] proved that for all $n \geq 0$,

$$
\begin{aligned}
& c_{8 n}, c_{8 n+1}, c_{8 n+2}>0, \quad c_{8 n+12}, c_{8 n+5}, c_{8 n+6}<0, \\
& d_{8 n}, d_{8 n+3}, d_{8 n+5}>0, \quad d_{8 n+1}, d_{8 n+4}, d_{8 n+7}<0 .
\end{aligned}
$$

In Section 3, using another approach, we prove a generalization of Hirschhorn's theorem:

Theorem 1.2. Suppose $m$ is divisible by 8 and $\operatorname{gcd}(m, r)=1$. Let $c_{n}$ and $d_{n}$ be defined by

$$
F_{m, 3 r, r}=\sum_{n=0}^{\infty} c_{n} q^{n} \quad \text { and } \quad F_{m, 3 r-m, r}=\sum_{n=0}^{\infty} d_{n} q^{n}
$$


Then, for all $n \geq 0$, we have

$$
\begin{array}{ll}
c_{8 n}, \quad c_{8 n+r}, \quad c_{8 n+2 r}>0, & c_{8 n+m-2 r}, \quad c_{8 n+m-3 r}<0, \\
d_{8 n}, d_{8 n+m-r}, d_{8 n+r}>0, & d_{8 n+4 r-m}, \quad d_{8 n+3 r-m}<0, \\
c_{8 n+m+4 r}<0 \text { if } m>8 r, & c_{8 n+2 m-4 r}<0 \text { if } m<8 r, \\
d_{8 n+5 r-m}<0 \text { if } 3 m>8 r, & d_{8 n+2 m-3 r}<0 \text { if } 3 m<8 r, \\
c_{n}=0 \text { if } n \equiv 3 r(\bmod 4) ; & d_{n}=0 \text { if } n \equiv 2 r(\bmod 4) .
\end{array}
$$

In Ramanujan's third notebook [1957, p. 373], there is a result equivalent to

$$
1+\frac{q+q^{2}}{1}+\frac{q^{2}+q^{4}}{1}+\cdots=F_{6,3,1} .
$$

In Section 4, we prove that the signs of the coefficients of $F_{6,3,1}$ are periodic with period 6 . This arises as a special case of the following theorem:

Theorem 1.3. Suppose $m$ is even and $\operatorname{gcd}(m, r)=1$. Let $c_{n}$ and $d_{n}$ be defined by

$$
F_{m, 3 r, r}=\sum_{n=0}^{\infty} c_{n} q^{n} \quad \text { and } \quad F_{m, 3 r-m, r}=\sum_{n=0}^{\infty} d_{n} q^{n} .
$$

Then, for all $n \geq m^{3}$, the signs of $c_{n}$ and $d_{n}$ are periodic with period at most $m$.

We conclude our paper with an interesting observation on the signs of the power series coefficients of the following continued fraction of Ramanujan:

$$
\frac{\left(q^{2} ; q^{3}\right)_{\infty}}{\left(q ; q^{3}\right)_{\infty}}=\frac{1}{1}-\frac{q}{1+q}-\frac{q^{3}}{1+q^{2}}-\frac{q^{5}}{1+q^{3}}-\cdots
$$

For a recent account of (6) see [Andrews et al. 2003].

\section{Definitions and Preliminary Results}

We first recall Ramanujan's definitions for a general theta function and some of its important special cases. Set

$$
f(a, b):=\sum_{n=-\infty}^{\infty} a^{n(n+1) / 2} b^{n(n-1) / 2}, \quad|a b|<1 .
$$

Basic properties satisfied by $f(a, b)$ include (see [Berndt 1991, p. 34, Entry 18]):

$$
f(a, b)=f(b, a), \quad f(1, a)=2 f\left(a, a^{3}\right), \quad f(-1, a)=0,
$$

and, if $l$ is an integer,

$$
f(a, b)=a^{l(l+1) / 2} b^{l(l-1) / 2} f\left(a(a b)^{l}, b(a b)^{-l}\right) .
$$


If $l=1,(8)$ reduces to

$$
f(a, b)=a f\left(a^{-1}, a^{2} b\right) .
$$

The function $f(a, b)$ satisfies the well-known Jacobi triple product identity (see [Berndt 1991, p. 35. Entry 19]):

$$
f(a, b)=(-a ; a b)_{\infty}(-b ; a b)_{\infty}(a b ; a b)_{\infty} .
$$

The three most important special cases of (7) are

$$
\begin{aligned}
\varphi(-q) & :=f(-q,-q)=\sum_{n=-\infty}^{\infty}(-1)^{n} q^{n^{2}}=\frac{(q ; q)_{\infty}}{(-q ; q)_{\infty}}, \\
\psi(q) & :=f\left(q, q^{3}\right) \quad=\sum_{n=0}^{\infty} q^{n(n+1) / 2}=\frac{\left(q^{2} ; q^{2}\right)_{\infty}}{\left(q ; q^{2}\right)_{\infty}}=\frac{(-q ;-q)_{\infty}}{\left(q^{2} ; q^{4}\right)_{\infty}}, \\
f(-q) & :=f\left(-q,-q^{2}\right)=\sum_{n=-\infty}^{\infty}(-1)^{n} q^{n(3 n-1) / 2}=(q ; q)_{\infty} .
\end{aligned}
$$

The product representations in these equalities are special cases of (10). Two further corollaries of (10) are given by

$$
\begin{aligned}
f(a, b) f(-a,-b) & =f\left(-a^{2},-b^{2}\right) \varphi(-a b), \\
f(a, b) \psi(a b) & =f\left(a, a b^{2}\right) f\left(b, b a^{2}\right) ;
\end{aligned}
$$

see [Berndt 1991, p. 46, Entry 30].

The well known quintuple product identity, in the notation of (7), takes the form

$$
\frac{f\left(-a^{2},-a^{-2} q\right)}{f\left(-a,-a^{-1} q\right)}=\frac{1}{f(-q)}\left(f\left(-a^{3} q,-a^{-3} q^{2}\right)+a f\left(-a^{-3} q,-a^{3} q^{2}\right)\right)
$$

see [Berndt 1991, p. 80, Entry 28(iv)].

We will need an expansion formula for $f(a, b)$. For each positive integer $l$, set

$$
U_{l}:=a^{l(l+1) / 2} b^{l(l-1) / 2} \quad \text { and } \quad V_{l}:=a^{l(l-1) / 2} b^{l(l+1) / 2} .
$$

Then, as in [Berndt 1991, p. 48, Entry 31], we have

$$
f\left(U_{1}, V_{1}\right)=\sum_{r=0}^{l-1} U_{r} f\left(\frac{U_{l+r}}{U_{r}}, \frac{V_{l-r}}{U_{r}}\right) .
$$

The special case of this for $l=2$ is

$$
f(a, b)=f\left(a^{3} b, a b^{3}\right)+a f\left(\frac{b}{a}, \frac{a}{b} a^{4} b^{4}\right) .
$$


Lastly, we record two elementary results [Berndt 1991, p. 45, Entry 29]. If $a b=c d$, then

$$
\begin{aligned}
& f(a, b) f(c, d)+f(-a,-b) f(-c,-d)=2 f(a c, b d) f(a d, b c) \\
& \begin{aligned}
f(a, b) f(c, d)-f(-a,-b) f(-c,-d)=2 a f\left(\frac{b}{c}, \frac{c}{b} a b c d\right) \\
\times f\left(\frac{b}{d}, \frac{d}{b} a b c d\right) .
\end{aligned}
\end{aligned}
$$

By adding (18) and (19), we conclude if $a b=c d$ then

$$
f(a, b) f(c, d)=f(a c, b d) f(a d, b c)+a f\left(\frac{b}{c}, \frac{c}{b} a b c d\right) f\left(\frac{b}{d}, \frac{d}{b} a b c d\right) .
$$

\section{Proof of Theorem 1.2}

The proof of Theorem 1.2 follows easily from the following lemma.

Lemma 3.1. For any $z \neq 0$ and $|q|<1$,

$$
\begin{aligned}
\frac{f\left(-z^{3},-z^{-3} q\right)}{f\left(-z,-z^{-1} q\right)}= & w_{1}(q) f\left(z^{8} q^{3}, z^{-8} q^{5}\right)+z^{2} w_{1}(q) f\left(z^{-8} q^{3}, z^{8} q^{5}\right) \\
& -q z^{-2} w_{2}(q) f\left(z^{8} q, z^{-8} q^{7}\right)-q z^{4} w_{2}(q) f\left(z^{-8} q, z^{8} q^{7}\right) \\
& +z w_{3}(q) f\left(z^{-8} q^{4}, z^{8} q^{4}\right)-q z^{-3} w_{3}(q) f\left(z^{8}, z^{-8} q^{8}\right)
\end{aligned}
$$

where

$$
w_{1}(q)=\frac{f\left(q^{3}, q^{5}\right)}{f(-q) f\left(-q^{2}\right)}, \quad w_{2}(q)=\frac{f\left(q, q^{7}\right)}{f(-q) f\left(-q^{2}\right)}, \quad w_{3}(q)=\frac{1}{\varphi(-q)}
$$

Proof. Let

$$
L(z, q)=\frac{f\left(-z^{3},-z^{-3} q\right)}{f\left(-z,-z^{-1} q\right)} .
$$

By (18) and (12), as a function of $z$, the even part of $L(z, q)$ is

$$
\begin{aligned}
L_{e}(z, q) & :=\frac{1}{2}\left(\frac{f\left(-z^{3},-z^{-3} q\right)}{f\left(-z,-z^{-1} q\right)}+\frac{f\left(z^{3}, z^{-3} q\right)}{f\left(z, z^{-1} q\right)}\right) \\
& =\frac{1}{2} \frac{f\left(-z^{3},-z^{-3} q\right) f\left(z, z^{-1} q\right)+f\left(z^{3}, z^{-3} q\right) f\left(-z,-z^{-1} q\right)}{f\left(-z,-z^{-1} q\right) f\left(z, z^{-1} q\right)} \\
& =\frac{f\left(-z^{4},-z^{-4} q^{2}\right) f\left(-z^{2} q,-z^{-2} q\right)}{f\left(-z^{2},-z^{-2} q^{2}\right) \varphi(-q)}
\end{aligned}
$$


By (13) and (12),

$$
\begin{aligned}
f\left(-z^{4},-z^{-4} q^{2}\right) \psi\left(q^{2}\right) & =f\left(-z^{4},-z^{-4} q^{4}\right) f\left(-z^{4} q^{2},-z^{-4} q^{2}\right) \\
& =\frac{f\left(-z^{2},-z^{-2} q^{2}\right) f\left(z^{2}, z^{-2} q^{2}\right) f\left(-z^{4} q^{2},-z^{-4} q^{2}\right)}{\varphi\left(-q^{2}\right)} .
\end{aligned}
$$

Employing (13) again, this time with $a=z^{2}$ and $b=-z^{-2} q$, we also obtain

$$
f\left(z^{2},-z^{-2} q\right) \psi(-q)=f\left(z^{2}, z^{-2} q^{2}\right) f\left(-z^{-2} q,-z^{2} q\right)
$$

We now return to the evaluation of the even part of $L(z, q)$. Using the last two displayed equations, we find that (23) is equivalent to

$$
L_{e}(z, q)=\frac{f\left(-z^{4} q^{2},-z^{-4} q^{2}\right) f\left(z^{2},-z^{-2} q\right) \psi(-q)}{\varphi(-q) \varphi\left(-q^{2}\right) \psi\left(q^{2}\right)} .
$$

But, by equations (11),

$$
\begin{aligned}
\frac{\varphi(-q) \varphi\left(-q^{2}\right) \psi\left(q^{2}\right)}{\psi(-q)} & =\frac{\frac{(q ; q)_{\infty}}{(-q ; q)_{\infty}} \frac{\left(q^{2} ; q^{2}\right)_{\infty}}{\left(-q^{2} ; q^{2}\right)_{\infty}} \frac{\left(q^{4} ; q^{4}\right)_{\infty}}{\left(q^{2} ; q^{4}\right)_{\infty}}}{\frac{(q ; q)_{\infty}}{\left(q^{2} ; q^{4}\right)_{\infty}}} \\
& =(q ; q)_{\infty}\left(q^{2} ; q^{2}\right)_{\infty}=f(-q) f\left(-q^{2}\right)
\end{aligned}
$$

Putting this together with (17) and (20), we find that (24) is transformed into

(25) $L_{e}(z, q)$

$$
\begin{aligned}
& =\frac{f\left(-z^{4} q^{2},-z^{-4} q^{2}\right) f\left(z^{2},-z^{-2} q\right)}{f(-q) f\left(-q^{2}\right)} \\
& =\frac{f\left(-z^{4} q^{2},-z^{-4} q^{2}\right)\left(f\left(-z^{-4} q,-z^{4} q^{3}\right)+z^{2} f\left(-z^{-4} q,-z^{4} q^{3}\right)\right)}{f(-q) f\left(-q^{2}\right)} \\
& =\frac{1}{f(-q) f\left(-q^{2}\right)}\left(f\left(-z^{4} q,-z^{-4} q^{3}\right) f\left(-z^{4} q^{2},-z^{-4} q^{2}\right)\right. \\
& \left.\quad+z^{2} f\left(-z^{-4} q,-z^{4} q^{3}\right) f\left(-z^{4} q^{2},-z^{-4} q^{2}\right)\right) \\
& =\frac{1}{f(-q) f\left(-q^{2}\right)}\left(f\left(z^{-8} q^{5}, z^{8} q^{3}\right) f\left(q^{3}, q^{5}\right)-z^{4} q f\left(z^{-8} q, z^{8} q^{7}\right) f\left(q, q^{7}\right)\right. \\
& \left.\quad+z^{2}\left(f\left(z^{-8} q^{3}, z^{8} q^{5}\right) f\left(q^{3}, q^{5}\right)-z^{-4} q f\left(z^{8} q, z^{-8} q^{7}\right)\right) f\left(q, q^{7}\right)\right) .
\end{aligned}
$$


As for the odd part of $L(z, q)$ as a function of $z$, we have, by (19), (12), and (17),

$$
\begin{aligned}
L_{o}(z, q) & :=\frac{1}{2}\left(\frac{f\left(-z^{3},-z^{-3} q\right)}{f\left(-z,-z^{-1} q\right)}-\frac{f\left(z^{3}, z^{-3} q\right)}{f\left(z, z^{-1} q\right)}\right) \\
& =\frac{1}{2} \frac{f\left(z, z^{-1} q\right) f\left(-z^{3},-z^{-3} q\right)-f\left(-z,-z^{-1} q\right) f\left(z^{3}, z^{-3} q\right)}{f\left(-z,-z^{-1} q\right) f\left(z, z^{-1} q\right)} \\
& =z \frac{f\left(-z^{-4} q,-z^{4} q\right) f\left(-z^{2},-z^{-2} q^{2}\right)}{f\left(-z^{2},-z^{-2} q^{2}\right) \varphi(-q)} \\
& =z \frac{f\left(-z^{-4} q,-z^{4} q\right)}{\varphi(-q)}=z \frac{f\left(z^{-8} q^{4}, z^{8} q^{4}\right)-q z^{-4} f\left(z^{8}, z^{-8} q^{8}\right)}{\varphi(-q)} .
\end{aligned}
$$

By adding (25) and (26), we complete the proof of Lemma 3.1.

We are now ready to prove Theorem 1.2. Observe that, by (10),

$$
F_{m, r, s}=\frac{f\left(-q^{r},-q^{m-r}\right)}{f\left(-q^{s},-q^{m-s}\right)} .
$$

Now assume that $3 r<m$. In (21), replace $q$ by $q^{m}$ and $z$ by $q^{r}$, respectively, to find that

(27) $F_{m, 3 r, r}$

$$
\begin{aligned}
= & \frac{f\left(-q^{3 r},-q^{m-3 r}\right)}{f\left(-q^{r},-q^{m-r}\right)} \\
= & w_{1}\left(q^{m}\right) f\left(q^{3 m+8 r}, q^{5 m-8 r}\right)+q^{2 r} w_{1}\left(q^{m}\right) f\left(q^{3 m-8 r}, q^{5 m+8 r}\right) \\
& -q^{m-2 r} w_{2}\left(q^{m}\right) f\left(q^{m+8 r}, q^{7 m-8 r}\right)-q^{m+4 r} w_{2}\left(q^{m}\right) f\left(q^{m-8 r}, q^{7 m+8 r}\right) \\
& +q^{r} w_{3}\left(q^{m}\right) f\left(q^{4 m-8 r}, q^{4 m+8 r}\right)-q^{m-3 r} w_{3}\left(q^{m}\right) f\left(q^{8 r}, q^{8 m-8 r}\right),
\end{aligned}
$$

where $w_{1}(q), w_{2}(q), w_{3}(q)$, defined in (22), are clearly power series in $q$ with positive coefficients. Since $3 r<m$, all the exponents in (27) are positive, except possibly those of $f\left(q^{m-8 r}, q^{7 m+8 r}\right)$. But by (9), we have

$$
f\left(q^{m-8 r}, q^{7 m+8 r}\right)=q^{m-8 r} f\left(q^{8 r-m}, q^{9 m-8 r}\right) .
$$

Thus the first part of (3) has been established.

Now suppose that $m / 3<r<m / 2$. We have, by (9),

$$
\frac{f\left(-q^{3 r},-q^{m-3 r}\right)}{f\left(-q^{r},-q^{m-r}\right)}=-q^{m-3 r} \frac{f\left(-q^{2 m-3 r},-q^{3 r-m}\right)}{f\left(-q^{r},-q^{m-r}\right)} .
$$


Thus, by (27) and by (9),

$$
\begin{aligned}
F_{m, 3 r-m, r}=-q^{3 r-m} & w_{1}\left(q^{m}\right) f\left(q^{3 m+8 r}, q^{5 m-8 r}\right)-q^{5 r-m} w_{1}\left(q^{m}\right) f\left(q^{3 m-8 r}, q^{5 m+8 r}\right) \\
& +q^{r} w_{2}\left(q^{m}\right) f\left(q^{m+8 r}, q^{7 m-8 r}\right)+q^{7 r} w_{2}\left(q^{m}\right) f\left(q^{m-8 r}, q^{7 m+8 r}\right) \\
& -q^{4 r-m} w_{3}\left(q^{m}\right) f\left(q^{4 m-8 r}, q^{4 m+8 r}\right)+w_{3}\left(q^{m}\right) f\left(q^{8 r}, q^{8 m-8 r}\right) \\
=-q^{3 r-m} & w_{1}\left(q^{m}\right) f\left(q^{3 m+8 r}, q^{5 m-8 r}\right)-q^{5 r-m} w_{1}\left(q^{m}\right) f\left(q^{3 m-8 r}, q^{5 m+8 r}\right) \\
& +q^{r} w_{2}\left(q^{m}\right) f\left(q^{m+8 r}, q^{7 m-8 r}\right)+q^{m-r} w_{2}\left(q^{m}\right) f\left(q^{8 r-m}, q^{9 m-8 r}\right) \\
& -q^{4 r-m} w_{3}\left(q^{m}\right) f\left(q^{4 m-8 r}, q^{4 m+8 r}\right)+w_{3}\left(q^{m}\right) f\left(q^{8 r}, q^{8 m-8 r}\right) .
\end{aligned}
$$

Since $m / 3<r<m / 2$, all the exponents in this expression are positive, except possibly those of $f\left(q^{3 m-8 r}, q^{5 m+8 r}\right)$. But by (9), we have

$$
f\left(q^{3 m-8 r}, q^{5 m+8 r}\right)=q^{3 m-8 r} f\left(q^{8 r-3 m}, q^{11 m-8 r}\right) .
$$

Hence the proof of Theorem 1.2 is complete.

\section{Proof of Theorem 1.3}

To prove Theorem 1.3, we need the following reformulation of Lemma 3.1.

\section{Lemma 4.1.}

$$
\frac{f\left(-z^{3},-z^{-3} q\right)}{f\left(-z,-z^{-1} q\right)}=\frac{1}{2}\left(\frac{f\left(z^{2}, z^{-2} \sqrt{q}\right)}{\psi(\sqrt{q})}+\frac{f\left(z^{2},-z^{-2} \sqrt{q}\right)}{\psi(-\sqrt{q})}\right)+z \frac{f\left(-z^{4} q,-z^{-4} q\right)}{\varphi(-q)} .
$$

Proof. Recall that $E_{e}(z, q)$ is defined by (23). By (26) and (25), it suffices to prove that

$$
L_{e}(z, q)=\frac{1}{2}\left(\frac{f\left(z^{2}, z^{-2} \sqrt{q}\right)}{\psi(\sqrt{q})}+\frac{f\left(z^{2},-z^{-2} \sqrt{q}\right)}{\psi(-\sqrt{q})}\right)
$$

To verify (29), we first recall that $\psi(q)$ is defined in (11). By (17),

$$
\begin{aligned}
\psi(q) & =f\left(q^{6}, q^{10}\right)+q f\left(q^{2}, q^{14}\right) \\
f\left(z, z^{-1} q\right) & =f\left(z^{2} q, z^{-2} q^{3}\right)+z f\left(z^{-2} q, z^{2} q^{3}\right) .
\end{aligned}
$$


Thus,

$$
\begin{aligned}
& \psi(-q) f\left(z, z^{-1} q\right)+\psi(q) f\left(z,-z^{-1} q\right) \\
& \begin{array}{r}
=\left(f\left(q^{6}, q^{10}\right)-q f\left(q^{2}, q^{14}\right)\right)\left(f\left(z^{2} q, z^{-2} q^{3}\right)+z f\left(z^{-2} q, z^{2} q^{3}\right)\right) \\
+\left(f\left(q^{6}, q^{10}\right)+q f\left(q^{2}, q^{14}\right)\right)\left(f\left(-z^{2} q,-z^{-2} q^{3}\right)+z f\left(-z^{-2} q,-z^{2} q^{3}\right)\right) \\
=f\left(q^{6}, q^{10}\right)\left(f\left(z^{2} q, z^{-2} q^{3}\right)+f\left(-z^{2} q,-z^{-2} q^{3}\right)\right) \\
+z f\left(q^{6}, q^{10}\right)\left(f\left(z^{-2} q, z^{2} q^{3}\right)+f\left(-z^{-2} q,-z^{2} q^{3}\right)\right) \\
-q z f\left(q^{2}, q^{14}\right)\left(f\left(z^{-2} q, z^{2} q^{3}\right)-f\left(-z^{-2} q,-z^{2} q^{3}\right)\right) \\
-q f\left(q^{2}, q^{14}\right)\left(f\left(z^{2} q, z^{-2} q^{3}\right)-f\left(-z^{2} q,-z^{-2} q^{3}\right)\right) .
\end{array}
\end{aligned}
$$

From (17), we have

$$
\begin{aligned}
& f(a, b)+f(-a,-b)=2 f\left(a^{3} b, a b^{3}\right) \\
& f(a, b)-f(-a,-b)=2 a f\left(\frac{b}{a}, \frac{a}{b} a^{4} b^{4}\right) .
\end{aligned}
$$

Employing this in (30), we find that

$$
\begin{aligned}
\psi(-q) f\left(z, z^{-1} q\right)+\psi(q) f\left(z,-z^{-1} q\right) \\
=2 f\left(q^{6}, q^{10}\right) f\left(z^{4} q^{6}, z^{-4} q^{10}\right)+2 z f\left(q^{6}, q^{10}\right) f\left(z^{-4} q^{6}, z^{4} q^{10}\right) \\
\quad-2 z^{-1} q f\left(q^{2}, q^{14}\right) f\left(z^{4} q^{2}, z^{-4} q^{14}\right)-2 z^{2} q^{2} f\left(q^{2}, q^{14}\right) f\left(z^{-4} q^{2}, z^{4} q^{14}\right)
\end{aligned}
$$

By the second line in (11),

$$
\begin{aligned}
\psi(-q) \psi(q) & =\frac{\left(q^{2} ; q^{2}\right)_{\infty}}{\left(q ; q^{2}\right)_{\infty}} \frac{(q ; q)_{\infty}}{\left(q^{2} ; q^{4}\right)_{\infty}} \\
& =\frac{\left(q^{2} ; q^{4}\right)_{\infty}\left(q^{4} ; q^{4}\right)_{\infty}}{\left(q ; q^{2}\right)_{\infty}} \frac{\left(q ; q^{2}\right)_{\infty}\left(q^{2} ; q^{2}\right)_{\infty}}{\left(q^{2} ; q^{4}\right)_{\infty}} \\
& =f\left(-q^{2}\right) f\left(-q^{4}\right) .
\end{aligned}
$$

In (31), we divide both sides by $2 \psi(-q) \psi(q)$, then replace $q^{2}$ by $q$ and $z$ by $z^{2}$. By using (32) in the resulting equation and after comparing it to (25), we complete the proof of (29).

We are now ready to prove Theorem 1.3. We assume that $m$ is not divisible by 8 since this case is covered by Theorem 1.2. Suppose first that $m=2 k, k$ is odd, $1 \leq r<2 k / 3$ and $\operatorname{gcd}(r, 2 k)=1$. In Lemma 4.1 , replace $q$ by $q^{2 k}$ and $z$ by $q^{r}$. We 
deduce that

$$
\begin{aligned}
F_{2 k, 3 r, r} & =\frac{f\left(-q^{3 r},-q^{2 k-3 r}\right)}{f\left(-q^{r},-q^{2 k-r}\right)} \\
& =\frac{1}{2}\left(\frac{f\left(q^{2 r}, q^{k-2 r}\right)}{\psi\left(q^{k}\right)}+\frac{f\left(q^{2 r},-q^{k-2 r}\right)}{\psi\left(-q^{k}\right)}\right)+q^{r} \frac{f\left(-q^{2 k+4 r},-q^{2 k-4 r}\right)}{\varphi\left(-q^{2 k}\right)} .
\end{aligned}
$$

Let

$$
E(q)=\sum_{n=0}^{\infty} e_{n} q^{n} \quad \text { and } \quad D(q)=\sum_{n=0}^{\infty} d_{n} q^{n}
$$

denote the even and the odd parts of $F_{2 k, 3 r, r}$. Clearly, by (33), since $r$ is odd, we have

$$
D(q)=q^{r} \frac{f\left(-q^{2 k+4 r},-q^{2 k-4 r}\right)}{\varphi\left(-q^{2 k}\right)}
$$

and

$$
E(q)=\frac{1}{2}\left(\frac{f\left(q^{2 r}, q^{k-2 r}\right)}{\psi\left(q^{k}\right)}+\frac{f\left(q^{2 r},-q^{k-2 r}\right)}{\psi\left(-q^{k}\right)}\right) .
$$

We will show that the signs of the coefficients $e_{n}$ and $d_{n}$ are periodic with period $k$. Define

$$
T(r):= \begin{cases}(k-1) / 2 & \text { if } 2 r<k \\ (k+1) / 2 & \text { if } 2 r>k\end{cases}
$$

Employing (16) with $l=k$, we find that

$$
\begin{aligned}
D(q) & =\frac{q^{r}}{\varphi\left(-q^{2 k}\right)} \sum_{n=0}^{k-1}(-1)^{n} q^{2 k n^{2}+4 n r} f\left(-q^{2 k^{2}(k+2 n)+4 r k},-q^{2 k^{2}(k-2 n)-4 r k}\right) \\
= & \sum_{n=0}^{k-1}(-1)^{n} q^{2 k n^{2}+(4 n+1) r} \frac{f\left(-q^{2 k^{2}(k+2 n)+4 r k},-q^{2 k^{2}(k-2 n)-4 r k}\right)}{\varphi\left(-q^{2 k}\right)} \\
= & \sum_{n=0}^{T(r)}(-1)^{n} q^{2 k n^{2}+(4 n+1) r} \frac{f\left(-q^{2 k^{2}(k+2 n)+4 r k},-q^{2 k^{2}(k-2 n)-4 r k}\right)}{\varphi\left(-q^{2 k}\right)} \\
& \quad+\sum_{n=T(r)+1}^{k-1}(-1)^{n} q^{2 k n^{2}+(4 n+1) r} \frac{f\left(-q^{2 k^{2}(k+2 n)+4 r k},-q^{2 k^{2}(k-2 n)-4 r k}\right)}{\varphi\left(-q^{2 k}\right)}
\end{aligned}
$$

from which, after an application of (9), we get 


$$
\begin{gathered}
D(q)=\sum_{n=0}^{T(r)}(-1)^{n} q^{2 k n^{2}+(4 n+1) r} \frac{f\left(-q^{2 k^{2}(k+2 n)+4 r k},-q^{2 k^{2}(k-2 n)-4 r k}\right)}{\varphi\left(-q^{2 k}\right)} \\
+\sum_{n=T(r)+1}^{k-1}(-1)^{n} q^{2 k n^{2}+(4 n+1) r+2 k^{2}(k-2 n)-4 r k} \\
\times \frac{f\left(-q^{6 k^{3}-4 k^{2} n-4 r k},-q^{2 k^{2}(2 n-k)+4 r k}\right)}{\varphi\left(-q^{2 k}\right)},
\end{gathered}
$$

All the exponents in (36) are positive, and a typical term has the form

$$
(-1)^{n} q^{2 b k+(4 n+1) r} \frac{f\left(-q^{2 \alpha k},-q^{4 k^{3}-2 \alpha k}\right)}{\varphi\left(-q^{2 k}\right)},
$$

where $b$ is a nonnegative integer and $\alpha$ is a positive integer with $\operatorname{gcd}(\alpha, 2 k)=1$. It is easily seen that $2 b k+(4 n+1) r$ is bounded by $k^{3} / 2$. Also, by (10),

$$
\begin{aligned}
\frac{f\left(-q^{\alpha},-q^{2 k^{2}-\alpha}\right)}{\varphi(-q)} & =\frac{\left(q^{\alpha} ; q^{2 k^{2}}\right)_{\infty}\left(q^{2 k^{2}-\alpha} ; q^{2 k^{2}}\right)_{\infty}\left(q^{2 k^{2}} ; q^{2 k^{2}}\right)_{\infty}}{\left(q, q^{2}\right)_{\infty}^{2}\left(q^{2} ; q^{2}\right)_{\infty}} \\
& =\frac{\left(q^{\alpha} ; q^{2 k^{2}}\right)_{\infty}\left(q^{2 k^{2}-\alpha} ; q^{2 k^{2}}\right)_{\infty}\left(q^{2 k^{2}} ; q^{2 k^{2}}\right)_{\infty}}{\left(q ; q^{2}\right)_{\infty}(q ; q)_{\infty}} \\
& =\left(q ; q^{2}\right)_{\infty}^{-1} \prod_{\substack{1 \leq t \leq 2 k^{2} \\
t \neq \alpha, 2 k^{2}-\alpha, 2 k^{2}}}\left(q^{t} ; q^{2 k^{2}}\right)_{\infty}^{-1}
\end{aligned}
$$

is clearly a power series in $q$ with strictly positive coefficients. Since the numbers $2 b k+(4 n+1) r$, for $0 \leq n \leq k-1$, are all distinct modulo $k$, we have established that the signs of the coefficients $d_{n}$ of $D(q)$ are periodic with period (at most) $k$ for all $n>k^{3} / 2$. Recall that in the statement of Theorem 1.3 we gave the larger bound $\mathrm{m}^{3}$ for the index of nonzero coefficients. We will not be precise for the right order because our proofs are constructive for all the cases that we consider throughout

the paper.
Next we examine $E(q)$, defined by (34). It suffices to look at $\frac{f\left(q^{2 r}, q^{k-2 r}\right)}{\psi\left(q^{k}\right)}$. By (16), with $l=k$, we have

$$
\begin{aligned}
& \frac{f\left(q^{2 r}, q^{k-2 r}\right)}{\psi\left(q^{k}\right)} \\
& \quad=\sum_{n=0}^{k-1} q^{k n^{2} / 2+(4 r-k) n / 2} \frac{f\left(q^{k^{2}(k+2 n) / 2+(4 r-k) k / 2}, q^{k^{2}(k-2 n) / 2-(4 r-k) k / 2}\right)}{\psi\left(q^{k}\right)} .
\end{aligned}
$$

One can easily check that the values of $k n^{2} / 2+(4 r-k) n / 2$, for $0 \leq n \leq k-1$, are all distinct modulo $k$. By arguing as in (36), we can assume that each of the quotients in the series above has the form $f\left(q^{\alpha k}, q^{k^{3}-\alpha k}\right) / \psi\left(q^{k}\right)$, where $\alpha$ is an 
odd positive integer with $\operatorname{gcd}(\alpha, k)=1$. We claim that when expanded as a power series, the even part of this expression has positive coefficients while the odd part has negative coefficients. To verify this, it suffices to show that

$$
\frac{f\left(-q^{\alpha}, q^{k^{2}-\alpha}\right)}{\psi(-q)}
$$

is a power series with positive terms. By (10), we have

$$
\begin{aligned}
& \frac{f\left(-q^{\alpha}, q^{k^{2}-\alpha}\right)}{\psi(-q)} \\
& =\frac{\left(q^{\alpha} ;-q^{k^{2}}\right)_{\infty}\left(-q^{k^{2}-\alpha} ; q^{k^{2}}\right)_{\infty}\left(-q^{k^{2}} ;-q^{k^{2}}\right)_{\infty}}{\left(q ; q^{4}\right)_{\infty}\left(q^{3} ; q^{4}\right)_{\infty}\left(q^{4} ; q^{4}\right)_{\infty}} \\
& =\frac{\left(q^{\alpha} ; q^{2 k^{2}}\right)_{\infty}\left(-q^{k^{2}+\alpha} ; q^{2 k^{2}}\right)_{\infty}\left(-q^{k^{2}-\alpha} ; q^{2 k^{2}}\right)_{\infty}}{\times\left(q^{2 k^{2}-\alpha} ; q^{2 k^{2}}\right)_{\infty}\left(-q^{k^{2}} ; q^{2 k^{2}}\right)_{\infty}\left(q^{2 k^{2}} ; q^{2 k^{2}}\right)_{\infty}} \\
& =\frac{f\left(q ; q^{2}\right)_{\infty}\left(q^{4} ; q^{4}\right)_{\infty}}{\left(q^{\alpha+k^{2}}, q^{k^{2}-\alpha}\right)\left(-q^{k^{2}} ; q^{2 k^{2}}\right)_{\infty}} \\
& \left.\left(q^{4} ; q^{4}\right)_{\infty}{ }^{2 k^{2}-\alpha} ; q^{2 k^{2}}\right)_{\infty}\left(q^{\alpha} ; q^{2 k^{2}}\right)_{\infty} \\
& =\frac{f\left(q^{\alpha+k^{2}}, q^{k^{2}-\alpha}\right)\left(-q^{2}\right)_{\infty}}{\left.\left(q^{4} ; q^{4}\right)_{\infty} q^{2}\right)_{\infty}} \prod_{\substack{1 \leq t \leq 2 k^{2}, t \text { odd } \\
t \neq \alpha, 2 k^{2}-\alpha}}\left(q^{t} ; q^{2 k^{2}}\right)_{\infty}^{-1}=: \sum_{n=0}^{\infty} j_{n} q^{n} .
\end{aligned}
$$

Observe that $j_{n}>0$ for all $n$ unless $\alpha=1$. If $\alpha=1$, then $j_{n}>0$ for all $n \geq 3$. From (38), we easily see that the coefficients $e_{n}$ of $E(q)$ are nonzero for $n>m^{3}$ and (34) together with (38) implies that their signs are also periodic with period $k$.

We conclude that if $k$ is odd and

$$
F_{2 k, 3 r, r}=\sum_{n=0}^{\infty} c_{n} q^{n},
$$

then $c_{n} \neq 0$ for all $n>m^{3}$ and the signs of $c_{n}$ 's are periodic with period $m$.

Next, we sketch a proof for the case $m=4 k, k$ is odd, $1 \leq r<4 k / 3$ and $\operatorname{gcd}(r, 4 k)=1$. In Lemma 4.1, replace $q$ by $q^{4 k}$ and $z$ by $q^{r}$. We deduce that

$$
\begin{aligned}
F_{4 k, 3 r, r} & =\frac{f\left(-q^{3 r},-q^{4 k-3 r}\right)}{f\left(-q^{r},-q^{4 k-r}\right)} \\
& =\frac{1}{2}\left(\frac{f\left(q^{2 r}, q^{2 k-2 r}\right)}{\psi\left(q^{2 k}\right)}+\frac{f\left(q^{2 r},-q^{2 k-2 r}\right)}{\psi\left(-q^{2 k}\right)}\right)+q^{r} \frac{f\left(-q^{4 k+4 r},-q^{4 k-4 r}\right)}{\varphi\left(-q^{4 k}\right)} .
\end{aligned}
$$


Let $E_{1}(q)$ and $D_{1}(q)$ denote the even and the odd parts of $F_{4 k, 3 r, r}$. By (33), and since $r$ is odd,

$$
\begin{aligned}
& D_{1}(q)=q^{r} \frac{f\left(-q^{4 k+4 r},-q^{4 k-4 r}\right)}{\varphi\left(-q^{4 k}\right)}, \\
& E_{1}(q)=\frac{1}{2}\left(\frac{f\left(q^{2 r}, q^{2 k-2 r}\right)}{\psi\left(q^{2 k}\right)}+\frac{f\left(q^{2 r},-q^{2 k-2 r}\right)}{\psi\left(-q^{2 k}\right)}\right) .
\end{aligned}
$$

We expand $D_{1}(q)$ by employing (16) with $l=k$, and by arguing as in (36)-(37), we find that the coefficients of $D_{1}(q)$ as a power series in $q$ are nonzero and their signs are periodic with period $k$, hence with period $2 k$ for $n>m^{3}$.

Next, we examine $E_{1}(q)$. Expanding each term of (40) by (16) with $l=k$, and arguing as in (38), we deduce that

$$
E_{1}(q)=\frac{1}{2} \sum_{n=0}^{k-1} q^{b_{n}}\left(\frac{f\left(q^{2 k \alpha_{n}}, q^{2 k^{3}-2 k \alpha_{n}}\right)}{\psi\left(q^{2 k}\right)}+\delta_{n} \frac{f\left(q^{2 k \alpha_{n}},-q^{2 k^{3}-2 k \alpha_{n}}\right)}{\psi\left(-q^{2 k}\right)}\right),
$$

where the $b_{n}, 0 \leq n<k$, are all distinct modulo $k$, each $\delta_{n}$ is 1 or -1 , each $\alpha_{n}$ is odd, and $\operatorname{gcd}\left(\alpha_{n}, k\right)=1$. We drop the index $n$, replace $q^{2 k}$ by $q$ and examine

$$
B(q):=\frac{f\left(q^{\alpha}, q^{k^{2}-\alpha}\right)}{\psi(q)} \mp \frac{f\left(q^{\alpha},-q^{k^{2}-\alpha}\right)}{\psi(-q)} .
$$

It suffices to show that the coefficients of the even and the odd part of $B(q)$ have constant signs. Let

$$
\frac{f\left(q^{\alpha}, q^{k^{2}-\alpha}\right)}{\psi(q)}=: \sum_{n=0}^{\infty}(-1)^{n} s_{n} q^{n} \quad \text { and } \quad \frac{f\left(q^{\alpha},-q^{k^{2}-\alpha}\right)}{\psi(-q)}=: \sum_{n=0}^{\infty} z_{n} q^{n} .
$$

It follows from (39) that $s_{n}>0$ for all $n \geq 3$ and by similar reasoning as in (39), one can show that $z_{n}>0$ for all $n \geq 3$. Therefore, it remains to show that the coefficients of

$$
\sum_{n=0}^{\infty}\left(s_{n}-z_{n}\right) q^{n}=\frac{f\left(-q^{\alpha}, q^{k^{2}-\alpha}\right)}{\psi(-q)}-\frac{f\left(q^{\alpha},-q^{k^{2}-\alpha}\right)}{\psi(-q)}
$$

have constant signs. But by (17), we have

$$
\begin{aligned}
\frac{1}{\psi(-q)}( & \left.f\left(-q^{\alpha}, q^{k^{2}-\alpha}\right)-f\left(q^{\alpha},-q^{k^{2}-\alpha}\right)\right) \\
= & \frac{1}{\psi(-q)}\left(f\left(-q^{k^{2}+2 \alpha},-q^{3 k^{2}-2 \alpha}\right)-q^{\alpha} f\left(-q^{k^{2}-2 \alpha},-q^{3 k^{2}+2 \alpha}\right)\right. \\
& \left.-f\left(-q^{k^{2}+2 \alpha},-q^{3 k^{2}-2 \alpha}\right)-q^{\alpha} f\left(-q^{k^{2}-2 \alpha},-q^{3 k^{2}+2 \alpha}\right)\right) \\
& =-2 q^{\alpha} \frac{f\left(-q^{k^{2}-2 \alpha},-q^{3 k^{2}+2 \alpha}\right)}{\psi(-q)} .
\end{aligned}
$$


Assuming without loss of generality that $k^{2}-2 \alpha>0$, we have, by (10),

$$
\begin{aligned}
\frac{f\left(-q^{k^{2}-2 \alpha},-q^{3 k^{2}+2 \alpha}\right)}{\psi(-q)} & =\frac{\left(q^{k^{2}-2 \alpha} ; q^{4 k^{2}}\right)_{\infty}\left(q^{3 k^{2}+2 \alpha} ; q^{4 k^{2}}\right)_{\infty}\left(q^{4 k^{2}} ; q^{4 k^{2}}\right)_{\infty}}{\left(q ; q^{4}\right)_{\infty}\left(q^{3} ; q^{4}\right)_{\infty}\left(q^{4} ; q^{4}\right)_{\infty}} \\
& =\frac{\left(q^{k^{2}-2 \alpha} ; q^{4 k^{2}}\right)_{\infty}\left(q^{3 k^{2}+2 \alpha} ; q^{4 k^{2}}\right)_{\infty}\left(q^{4 k^{2}} ; q^{4 k^{2}}\right)_{\infty}}{\left(q^{4} ; q^{4}\right)_{\infty}\left(q ; q^{2}\right)_{\infty}} \\
& =\prod_{1 \leq t<k^{2}}\left(q^{4 t} ; q^{4 k^{2}}\right)_{\infty}^{-1} \prod_{\substack{1 \leq t \leq 4 k^{2}, t o d d \\
t \neq k^{2}-2 \alpha, 3 k^{2}+2 \alpha}}\left(q^{t} ; q^{4 k^{2}}\right)_{\infty}^{-1}
\end{aligned}
$$

Denoting by $\sum_{n=0}^{\infty} u_{n} q^{n}$ the corresponding power series, we see that $u_{n}>0$ for all $n \geq 3$. Thus, we have shown that the coefficients $E_{1}(q)$ as a power series in $q$ are nonzero and their signs are periodic with period $2 k$ for $n>m^{3}$.

Arguing as in (28), one can also establish periodicity modulo $m$ for the signs of the coefficients of $F_{m, 3 r-m, r}$.

As a special case, we consider Ramanujan's cubic continued fraction, defined by (4).

Corollary 4.2. Let

$$
\frac{f\left(-q^{3},-q^{3}\right)}{f\left(-q,-q^{5}\right)}=\sum_{n=0}^{\infty} c_{n} q^{n} .
$$

Then

$$
\begin{aligned}
& c_{6 n+1}>0, \quad c_{6 n+3}<0, \quad c_{6 n+11}<0 \quad \text { for all } n \geq 0, \\
& c_{6 n}>0, \quad c_{6 n+4}<0, \quad c_{6 n+14}>0 \quad \text { for all } n \geq 0 .
\end{aligned}
$$

Proof. By Lemma 4.1,

$$
\frac{f\left(-q^{3},-q^{3}\right)}{f\left(-q,-q^{5}\right)}=\frac{1}{2}\left(\frac{f\left(q, q^{2}\right)}{\psi\left(q^{3}\right)}+\frac{f\left(-q, q^{2}\right)}{\psi\left(-q^{3}\right)}\right)+q \frac{f\left(-q^{2},-q^{10}\right)}{\varphi\left(-q^{6}\right)} .
$$

By (10), and some elementary product manipulations one can actually show that

$$
\frac{f\left(-q^{3},-q^{3}\right)}{f\left(-q,-q^{5}\right)}=\frac{f\left(q, q^{2}\right)}{\psi\left(q^{3}\right)} .
$$

Employing (16) with $l=3, a$ replaced by $-q^{2}$ and $b$ replaced by $-q^{10}$, we obtain

$$
\begin{aligned}
& q \frac{f\left(-q^{2},-q^{10}\right)}{\varphi\left(-q^{6}\right)} \\
& \quad=\frac{q}{\varphi\left(-q^{6}\right)}\left(f\left(-q^{42},-q^{66}\right)-q^{2} f\left(-q^{30},-q^{78}\right)-q^{10} f\left(-q^{6},-q^{102}\right)\right),
\end{aligned}
$$


from which we deduce (41). Similarly, using (16) with $l=3$, we find that

$$
\frac{f\left(q, q^{2}\right)}{\psi\left(q^{3}\right)}=\frac{1}{\psi\left(q^{3}\right)}\left(f\left(q^{12}, q^{15}\right)+q f\left(q^{6}, q^{21}\right)+q^{2} f\left(q^{3}, q^{24}\right)\right)
$$

which yields (42).

\section{A generalization of Ramanathan's result}

In this section, we establish an expansion of $F_{m, 2 r, r}$ modulo $m$. Ramanathan determined the signs of the Taylor series coefficients of $F_{m, 2 r, r}$, under the assumption that $\operatorname{gcd}(6, m)=1$. His proof is similar to that of Andrews and uses Gordon's theorem [1961].

Theorem 5.1. If $m \equiv 1(\bmod 3)$, then

$$
\begin{aligned}
& \frac{f\left(-z^{2},-z^{-2} q\right)}{f\left(-z,-z^{-1} q\right)}=\frac{f\left(-q^{m^{2}}\right)}{f(-q)} \\
& \quad \times \sum_{n=0}^{m-1}(-1)^{n} z^{3 n} q^{n(3 n-1) / 2} \frac{f\left(-z^{2 m} q^{m(m+6 n-1) / 3},-z^{-2 m} q^{m(2 m-6 n+1) / 3}\right)}{f\left((-z)^{m} q^{m(m+6 n-1) / 6},(-z)^{-m} q^{m(5 m-6 n+1) / 6}\right)}
\end{aligned}
$$

If $m \equiv-1(\bmod 3)$, then

$$
\begin{aligned}
& \frac{f\left(-z^{2},-z^{-2} q\right)}{f\left(-z,-z^{-1} q\right)}=\frac{f\left(-q^{m^{2}}\right)}{f(-q)} \\
& \quad \times \sum_{n=0}^{m-1}(-1)^{n} z^{3 n} q^{n(3 n-1) / 2} \frac{f\left(-z^{-2 m} q^{m(m-6 n+1) / 3},-z^{2 m} q^{m(2 m+6 n-1) / 3}\right)}{f\left((-z)^{-m} q^{m(m-6 n+1) / 6},(-z)^{m} q^{m(5 m+6 n-1) / 6}\right)}
\end{aligned}
$$

If $m \equiv 0(\bmod 3)$, then

$$
\begin{aligned}
& \frac{f\left(-z^{2},-z^{-2} q\right)}{f\left(-z,-z^{-1} q\right)}=\frac{1}{f(-q)} \\
& \times\left(\sum_{n=0}^{m / 3-1}(-1)^{n} z^{3 n} q^{n(3 n-1) / 2} f\left((-z)^{m} q^{m(m+6 n-1) / 6},(-z)^{-m} q^{m(m-6 n+1) / 6}\right)\right. \\
& \left.+\sum_{t=0}^{m / 3-1}(-1)^{t} z^{1-3 t} q^{t(3 t-1) / 2} f\left((-z)^{-m} q^{m(m+6 t-1) / 6},(-z)^{m} q^{m(m-6 t+1) / 6}\right)\right) .
\end{aligned}
$$


Proof. We give proofs of (43) and (45). We will not prove (44) since its proof is very similar to that of (43). Let us assume first that $m$ is odd. By (14) and (16),

$$
\begin{aligned}
& \text { (46) } \frac{f\left(-z^{2},-z^{-2} q\right)}{f\left(-z,-z^{-1} q\right)} \\
& =\frac{1}{f(-q)}\left(f\left(-z^{3} q,-z^{-3} q^{2}\right)+z f\left(-z^{-3} q,-z^{3} q^{2}\right)\right) \\
& =\frac{1}{f(-q)}\left(\sum_{n=0}^{m-1}(-1)^{n} z^{3 n} q^{n(3 n-1) / 2} f\left(-z^{3 m} q^{m(3 m+6 n-1) / 2},-z^{-3 m} q^{m(3 m-6 n+1) / 2}\right)\right.
\end{aligned}
$$

$$
\left.+\sum_{t=0}^{m-1}(-1)^{n} z^{-3 t+1} q^{t(3 t-1) / 2} f\left(-z^{-3 m} q^{m(3 m+6 t-1) / 2},-z^{3 m} q^{m(3 m-6 t+1) / 2}\right)\right) .
$$

Employing (8) with $l=k$, we find that

$$
\begin{aligned}
f\left(-z^{-3 m} q^{m(3 m+6 t-1) / 2}\right. & \left.-z^{3 m} q^{m(3 m-6 t+1) / 2}\right)=(-1)^{k} z^{-3 m k} q^{m k(3 m k+6 t-1) / 2} \\
& \times f\left(-z^{-3 m} q^{m(3 m+6 m k+6 t-1) / 2},-z^{3 m} q^{m(3 m-6 m k-6 t+1) / 2}\right) .
\end{aligned}
$$

Assume now that $m \equiv 1(\bmod 3)$ and fix $n$ and $t$ so that $3 n \equiv-3 t+1(\bmod m)$. Thus, $3 n+3 t-1=(3 v-1) m$ for some integer $v$. By taking $k=-v$ in the preceding displayed equality and using the resulting equation in (46) we conclude after some elementary algebraic manipulation that

$$
\begin{aligned}
& \frac{f\left(-z^{2},-z^{-2} q\right)}{f\left(-z,-z^{-1} q\right)} \\
& =\frac{1}{f(-q)} \sum_{n=0}^{m-1}(-1)^{n} z^{3 n} q^{n(3 n-1) / 2}\left(f\left(-z^{3 m} q^{m(3 m+6 n-1) / 2},-z^{-3 m} q^{m(3 m-6 n+1) / 2}\right)\right. \\
& \left.\quad+z^{m} q^{m(m+6 r-1) / 6} f\left(-z^{-3 m} q^{m(m-6 n+1) / 2},-z^{3 m} q^{m(5 m+6 n-1) / 2}\right)\right) .
\end{aligned}
$$

Employing (14) again, this time with $a=z^{m} q^{m(m+6 r-1) / 6}$ and $q$ replaced by $q^{m^{2}}$, we find that

$$
\begin{aligned}
& \frac{f\left(-z^{-2 m} q^{m(m-6 n+1) / 3},-z^{2 m} q^{m(2 m+6 n-1) / 3}\right)}{f\left(-z^{-m} q^{m(m-6 n+1) / 6},-z^{m} q^{m(5 m+6 n-1) / 6}\right)} \\
& \quad=\frac{1}{f\left(-q^{m^{2}}\right)}\left(f\left(-z^{3 m} q^{m(3 m+6 n-1) / 2},-z^{-3 m} q^{m(3 m-6 n+1) / 2}\right)\right. \\
& \left.\quad+z^{m} q^{m(m+6 r-1) / 6} f\left(-z^{-3 m} q^{m(m-6 n+1) / 2},-z^{3 m} q^{m(5 m+6 n-1) / 2}\right)\right) .
\end{aligned}
$$

Substituting this equation in the preceding one, we see that the proof of (43) for the case of odd $m$ is complete. 
Now assume that $m$ is even. By (14) and (16),

$$
\begin{aligned}
& \text { (47) } \frac{f\left(-z^{2},-z^{-2} q\right)}{f\left(-z,-z^{-1} q\right)} \\
& =\frac{1}{f(-q)}\left(f\left(-z^{3} q,-z^{-3} q^{2}\right)+z f\left(-z^{-3} q,-z^{3} q^{2}\right)\right)
\end{aligned}
$$$$
=\frac{1}{f(-q)}\left(\sum_{n=0}^{m-1}(-1)^{n} z^{3 n} q^{n(3 n-1) / 2} f\left(z^{3 m} q^{m(3 m+6 n-1) / 2}, z^{-3 m} q^{m(3 m-6 n+1) / 2}\right)\right.
$$$$
\left.+\sum_{t=0}^{m-1}(-1)^{t} z^{-3 t+1} q^{t(3 t-1) / 2} f\left(z^{-3 m} q^{m(3 m+6 t-1) / 2}, z^{3 m} q^{m(3 m-6 t+1) / 2}\right)\right) .
$$

Employing (8) again with $l=k$, we find that

$$
\begin{aligned}
& f\left(z^{-3 m} q^{m(3 m+6 t-1) / 2}, z^{3 m} q^{m(3 m-6 t+1) / 2}\right) \\
& \quad=z^{-3 m k} q^{m k(3 m k+6 t-1) / 2} f\left(z^{-3 m} q^{m(3 m+6 m k+6 t-1) / 2}, z^{3 m} q^{m(3 m-6 m k-6 t+1) / 2}\right) .
\end{aligned}
$$

Fix $n$ and $t$ so that $3 n \equiv-3 t+1(\bmod m)$. Thus, $3 n+3 t-1=(3 v-1) m$ for some integer $v$. Since $m$ is even, we know that $n$ and $t$ have opposite parity. By taking $k=-v$ in the last displayed equality and then using the resulting equation in (47), we conclude this time that

$$
\begin{aligned}
& \frac{f\left(-z^{2},-z^{-2} q\right)}{f\left(-z,-z^{-1} q\right)} \\
& =\frac{1}{f(-q)} \sum_{n=0}^{m-1}(-1)^{n} z^{3 n} q^{n(3 n-1) / 2}\left(f\left(z^{3 m} q^{m(3 m+6 n-1) / 2}, z^{-3 m} q^{m(3 m-6 n+1) / 2}\right)\right. \\
& \left.\quad-z^{m} q^{m(m+6 r-1) / 6} f\left(z^{-3 m} q^{m(m-6 n+1) / 2}, z^{3 m} q^{m(5 m+6 n-1) / 2}\right)\right) .
\end{aligned}
$$

Employing (14) again this time with $a=-z^{m} q^{m(m+6 r-1) / 6}$ and $q$ replaced by $q^{m^{2}}$, we find that

$$
\begin{aligned}
& \frac{f\left(-z^{-2 m} q^{m(m-6 n+1) / 3},-z^{2 m} q^{m(2 m+6 n-1) / 3}\right)}{f\left(z^{-m} q^{m(m-6 n+1) / 6}, z^{m} q^{m(5 m+6 n-1) / 6}\right)} \\
& =\frac{1}{f\left(-q^{m^{2}}\right)}\left(f\left(z^{3 m} q^{m(3 m+6 n-1) / 2}, z^{-3 m} q^{m(3 m-6 n+1) / 2}\right)\right. \\
& \left.\quad-z^{m} q^{m(m+6 r-1) / 6} f\left(z^{-3 m} q^{m(m-6 n+1) / 2}, z^{3 m} q^{m(5 m+6 n-1) / 2}\right)\right) .
\end{aligned}
$$

This proves (43) for $m$ even.

Observe that (45) is just (46) or (47) with $m$ replaced by $m / 3$.

As a corollary of Theorem 5.1, we obtain a generalization of Theorem 1.1. 
Corollary 5.2. Suppose that $\operatorname{gcd}(r, m)=1$. Set

$$
F_{m, 2 r, r}:=\sum_{n=0}^{\infty} c_{n} q^{n} .
$$

If $m \neq \equiv 0(\bmod 3)$, then $c_{n} \neq 0$ for $n>3 m^{3}$ and the signs of the $c_{n}$ 's are periodic with period $m$.

If $m \equiv 0(\bmod 3)$, then $c_{n} \neq 0$ for $n>3 m^{3}$ and the signs of the $c_{n}$ 's are periodic with period $m$ unless $n \equiv-r(\bmod 3)$, in which case $c_{n} \equiv 0$.

Proof. We will sketch a proof for the case $m \equiv 4(\bmod 6)$. By (43) with $q$ replaced by $q^{m}$ and $z$ replaced by $q^{r}$, we find that

$$
\begin{aligned}
& F_{m, 2 r, r}=\frac{f\left(-q^{2 r},-q^{m-2 r}\right)}{f\left(-q^{r},-q^{m-r}\right)} \\
&=\frac{f\left(-q^{m^{3}}\right)}{f\left(-q^{m}\right)} \sum_{n=0}^{m-1}\left((-1)^{n} q^{m n(3 n-1) / 2+3 r n}\right. \\
&\left.\frac{f\left(-q^{m^{2}(m+6 n-1) / 3+2 m r},-q^{m^{2}(2 m-6 n+1) / 3-2 m r}\right)}{f\left(q^{m^{2}(m+6 n-1) / 6+m r}, q^{m^{2}(5 m-6 n+1) / 6-m r}\right)}\right) .
\end{aligned}
$$

We break up the summation into three parts and apply (9) if there are any negative exponents. We deduce that

$$
\begin{aligned}
& F_{m, 2 r, r}=\frac{f\left(-q^{m^{3}}\right)}{f\left(-q^{m}\right)} \\
& \times\left(\sum_{n=0}^{(m-1) / 3}(-1)^{n} q^{A(n)} \frac{f\left(-q^{m^{2}(m+6 n-1) / 3+2 m r},-q^{m^{2}(2 m-6 n+1) / 3-2 m r}\right)}{f\left(q^{m^{2}(m+6 n-1) / 6+m r}, q^{m^{2}(5 m-6 n+1) / 6-m r}\right)}\right. \\
& \quad+\sum_{n=(m+2) / 3}^{(5 m-2) / 6}\left((-1)^{n+1} q^{B(n)}\right. \\
& \left.\quad \times \frac{f\left(-q^{m^{2}(5 m-6 n+1) / 3-2 m r},-q^{m^{2}(6 n-2 m-1) / 3+2 m r}\right)}{f\left(q^{m^{2}(m+6 n-1) / 6+m r}, q^{m^{2}(5 m-6 n+1) / 6-m r}\right)}\right) \\
& \quad \sum_{n=(5 m+4) / 6}\left((-1)^{n} q^{C(n)}\right. \\
& \left.\left.\times \frac{f\left(-q^{m^{2}(6 n-5 m-1) / 3+2 m r},-q^{m^{2}(8 m-6 n+1) / 3-2 m r}\right)}{f\left(q^{m^{2}(11 m-6 n+1) / 6-m r}, q^{m^{2}(6 n-5 m-1) / 6+m r}\right)}\right)\right),
\end{aligned}
$$

where

$$
\begin{aligned}
& A(n)=m n(3 n-1) / 2+3 r n, \\
& B(n)=A(n)+m^{2}(2 m-6 n+1) / 3-2 m r, \\
& C(n)=B(n)+m^{2}(5 m-6 n+1) / 6-m r .
\end{aligned}
$$


Clearly $A(n) \equiv B(n) \equiv C(n)(\bmod m)$ and the $A(n)$, for $0 \leq n<m$, are all distinct modulo $m$. Each quotient of (48) upon replacing $q^{m}$ by $q$ has the form

$$
\frac{f\left(-q^{2 \alpha},-q^{m^{2}-2 \alpha}\right) f\left(-q^{m^{2}}\right)}{f\left(q^{\alpha}, q^{m^{2}-\alpha}\right) f(-q)},
$$

where $\operatorname{gcd}(\alpha, m)=1$. By employing (10) and some elementary product manipulations, we see that

$$
\begin{aligned}
& \frac{f\left(-q^{2 \alpha},-q^{m^{2}-2 \alpha}\right) f\left(-q^{m^{2}}\right)}{f\left(q^{\alpha},-q^{m^{2}-\alpha}\right) f(-q)} \\
& =\frac{1}{\left(q^{2 \alpha} ; q^{2 m^{2}}\right)_{\infty}\left(q^{2 m^{2}-2 \alpha} ; q^{2 m^{2}}\right)_{\infty}} \prod_{\substack{t \neq 2 \alpha, m^{2}-2 \alpha, \alpha, m^{2}-\alpha, m^{2} \\
t \neq t \leq m^{2}}}\left(q^{t} ; q^{m^{2}}\right)_{\infty}^{-1}
\end{aligned}
$$

is a power series in $q$ with strictly positive coefficients except possibly that of $q$. Since $A(n), B(n)$ and $C(n)$ are bounded by $2 m^{3}$, we conclude that the coefficients $c_{n}$ of $F_{m, 2 r, r}$ are nonzero and their signs are periodic modulo $m$ for $n>3 m^{3}$.

\section{The coefficients of a certain "non-theta" product}

Let $\beta_{n}$ be defined by

$$
\sum_{n=0}^{\infty} \beta_{n} q^{n}:=\frac{\left(q^{2} ; q^{3}\right)_{\infty}}{\left(q ; q^{3}\right)_{\infty}}=\frac{1}{1}-\frac{q}{1+q}-\frac{q^{3}}{1+q^{2}}-\frac{q^{5}}{1+q^{3}}-\ldots
$$

This continued fraction of Ramanujan differs from the other continued fractions considered in this paper because it is not a ratio of two theta functions. Using Maple, we computed $\beta_{n}$ for $0 \leq n \leq 1000$. In this range $\beta_{n}$ is nonzero except for $n=2,3,5,10,13,32$, and 80 . As for the sign of $\beta_{n}$, beginning at 63 , the signs have period 3 in intervals of increasing lengths. Thus, beginning at 63, the signs are,,-+- . At 82 , a new sequence,,++- starts . Then at 103 , the sequence,,-+begins, etc. Based on numerical evidence, we conjecture that the lengths of the intervals are strictly increasing therefore the signs of $\beta_{n}$ are not periodic. We are led to examine the series $\sum_{n=0}^{\infty} \beta_{3 n+j} q^{n}$ for $0 \leq j<2$. Here the signs are evidently constant in intervals of increasing lengths. For example, for $j=0$, starting at $n=2$, the lengths of the first six intervals are 5, 14, 21, 28, 36, and 43. We are unable to use the methods of this paper to examine the product in (49). Evidently, entirely new ideas will need to be devised to establish the observed phenomena.

\section{Acknowledgment}

We thank our adviser, Professor Bruce C. Berndt, for his guidance and assistance at all stages of this work. 


\section{References}

[Andrews 1981] G. E. Andrews, "Ramunujan's "lost" notebook, III: The Rogers-Ramanujan continued fraction”, Adv. in Math. 41:2 (1981), 186-208. MR 83m:10034c Zbl 0477.33009

[Andrews and Bressoud 1979] G. E. Andrews and D. M. Bressoud, "Vanishing coefficients in infinite product expansions”, J. Austral. Math. Soc. Ser. A 27:2 (1979), 199-202. MR 81f:10059 Zbl 0397.10047

[Andrews et al. 2003] G. E. Andrews, B. C. Berndt, J. Sohn, A. J. Yee, and A. Zaharescu, "On Ramanujan's continued fraction for $\left(q^{2} ; q^{3}\right)_{\infty} /\left(q ; q^{3}\right)_{\infty}$ ", Trans. Amer. Math. Soc. 355:6 (2003), 2397-2411. MR 2004d:11012 Zbl 1063.33026

[Berndt 1991] B. C. Berndt, Ramanujan's notebooks, III, Springer, New York, 1991. MR 92j:01069 Zbl 0733.11001

[Gordon 1961] B. Gordon, "A combinatorial generalization of the Rogers-Ramanujan identities", Amer. J. Math. 83 (1961), 393-399. MR 23 \#A809 Zbl 0100.27303

[Hardy and Wright 1960] G. H. Hardy and E. M. Wright, An introduction to the theory of numbers, 4th ed., Clarendon Press, Oxford, 1960. MR 81i:10002 Zbl 0086.25803

[Hirschhorn 1998] M. D. Hirschhorn, "On the expansion of Ramanujan's continued fraction", Ramanujan J. 2:4 (1998), 521-527. MR 1665326 (99i:11006) Zbl 0924.11005

[Hirschhorn 2001] M. D. Hirschhorn, "On the expansion of a continued fraction of Gordon", Ramanujan J. 5:4 (2001), 369-375. MR 2003a:11015 Zbl 0993.30003

[Ramanathan 1988] K. G. Ramanathan, "Generalisations of some theorems of Ramanujan", J. Number Theory 29:2 (1988), 118-137. MR 89k:11031 Zbl 0645.10026

[Ramanujan 1957] S. Ramanujan, Notebooks (2 vols.), Tata Institute of Fundamental Research, Bombay, 1957. Zbl 0138.24201

[Ramanujan 1988] S. Ramanujan, The lost notebook and other unpublished papers, Narosa, New Delhi, and Springer, Berlin, 1988. MR 89j:01078 Zbl 0639.01023

[Richmond and Szekeres 1978] B. Richmond and G. Szekeres, "The Taylor coefficients of certain infinite products", Acta Sci. Math. (Szeged) 40:3-4 (1978), 347-369. MR 80g:10046 Zbl 0397.10046

Received July 27, 2004. Revised 3/2/05.

\section{SONG HENG CHAN}

DEPARTMENT OF MATHEMATICS

NATIONAL UNIVERSITY OF SINGAPORE

2, SCIENCE DRIVE 2

SINGAPORE 117543

SINGAPORE

matcshs@nus.edu.sg

HAMZA YesilyurT

DEPARTMENT OF MATHEMATICS

UNIVERSITY OF FLORIDA

358 LITTLE HALL

GAINESVILLE, FL 32611-8105

UNITED STATES

hamza@math.ufl.edu 